\title{
Estrategia de reflexión para enseñanza de proyectos de construcción en Ingeniería Civil
}

\section{Reflection strategy for teaching construction projects in Civil Engineering}

\begin{abstract}
Albeiro Bejarano Castellanos es Arquitecto de Jaramillo Mora S.A. Cali (Colombia) (albeca34@hotmail.com) (https://orcid.org/0000-0003-3903-3099)
\end{abstract}

Dra. María Fernanda Serrano Guzmán es profesora titular de la Pontificia Universidad Javeriana Cali (Colombia), (maria.serrano@javerianacali.edu.co) (https://orcid.org/0000-0002-7366-6597)

Dr. Diego Darío Pérez Ruiz es profesor titular de la Pontificia Universidad Javeriana Cali (Colombia), ddperez@javerianacali.edu.co) (https://orcid.org/0000-0002-9656-2803)

Recibido: 2018-05-20 / Revisado: 2018-11-20 / Aceptado: 2018-11-15 / Publicado: 2019-01-01

\section{Resumen}

La competitividad de los programas educativos de las universidades exige que los contenidos de los programas académicos se ajusten de manera que puedan responder a las problemáticas del entorno. Por esta razón, en lo concerniente al perfil del desempeño ocupacional del ingeniero civil, en lo particular, resulta útil que el estudiante identifique prácticas y procesos administrativos que propendan por la reducción de costos y tiempos en las obras. En este artículo se presenta una propuesta de estrategias didácticas centradas en la reflexión alrededor de conceptos relacionados con administración de proyectos de construcción empleando reingeniería y la filosofía de construcción sin pérdidas (Lean) en diferentes actividades. Para ello, se parte de información recolecta- da en proyectos de construcción de vivienda en donde se evaluaron las mejores prácticas para la disminución de desperdicios. Se conjuga los conocimientos técnicos con herramientas centradas en la reflexión para afianzar conceptos de administración de recursos, tanto humanos como financieros, mediante herramientas que emplean pensamiento crítico, solución de problemas, compromiso social, trabajo en equipo y habilidades comunicativas las cuales, en conjunto, contribuyen de manera transversal a fortalecer aspectos éticos y de la responsabilidad social del ejercicio de la ingeniería. Se concluye del estudio que la reflexión es una herramienta útil del proceso de enseñanza-aprendizaje, ya que prepara al futuro profesional a resolver problemáticas de su entorno.

Forma sugerida de citar: Serrano Guzmán, M. F., Pérez Ruiz, D. D., \& Bejarano Castellanos, A. (2019). Estrategia de reflexión para enseñanza de proyectos de construcción en Ingeniería Civil. Alteridad, 14(1), 122-137. https://doi. org/10.17163/alt.v14n1.2019.10 
Descriptores: Aprendizaje, construcción, organización, competencias, trabajo de campo, vida profesional.

\begin{abstract}
The competitiveness of the educational programs on the universities requires an adjustment on the content of academic programs so that they can respond to the problems of their surroundings. For this reason, in regards to the profile of a civil engineer's occupational performance, particularly, it is useful for the student to identify administrative practices and processes that tend to reduce costs and time over the different works. In this work, it is presented a proposal of didactic strategies focused on the inside thought around concepts related to the administration of construction projects using reengineering and construction
\end{abstract}

\section{Introducción}

El currículum de Ingeniería Civil en las universidades incluye en la oferta académica cursos que en su contenido abordan temas relacionados con administración de obras, proyectos de construcción y procesos constructivos, entre otros, con los cuales se pretende aportar destrezas al futuro profesional para desempeñarse en el sector de la construcción. Comúnmente en el aula de clase, ambiente en donde confluyen diferentes aprendices con experiencias y preconceptos diversos (Ucol-Ganiron Jr. \& Alaboodi, 2013), se emplean diversas estrategias educativas que permiten la formación de un pensamiento crítico y reflexivo para profesionales (Arratia Figueroa, 2008) orientados al logro del bien común y hacia una sociedad más justa y democrática (Palomer, Humeres, Sánchez, González, \& Contreras, 2013). Usualmente, durante el desempeño ocupacional, el ingeniero debe emplear sus conocimientos para resolver problemas relacionados con procesos constructivos.

Por lo anterior, resulta imperativo el desarrollo de estrategias didácticas basadas en la reflexión sobre habilidades transversales que debe adquirir o desarrollar el estudiante sobre el compromiso social, pensamiento crítico, resolu- philosophy without losses (lean) concepts in different activities. For this, we started with information collected in housing construction projects, in which the best practices for waste reduction were evaluated. The applicability of technical knowledge is combined with tools focused on reflection to strengthen concepts of resource management, both human and financial, using tools that use critical thinking, problem solving, social commitment, teamwork and communication skills, which, all together, contribute to strengthen ethical aspects and social responsibility of the engineering practice. It is concluded from the study that inside though is a useful tool of the teaching-learning process, since it prepares the future professional to solve the problems surrounding it.

Keywords: Learning, construction, arrangement, skills development, fieldwork, working life.

ción de problemas y habilidades comunicativas de manera que el estudiante enfrente preconcepciones o prejuicios y al mismo tiempo se anticipe ante posibles imprevistos (Montalva, Ponce, y Llorca, 2017; Erren, 2008) que se pueden presentar durante la formulación o la ejecución de un proyecto, todo esto bajo la premisa que un buen profesional no solo es aquel que maneja el conocimiento teórico o técnico sino el que demuestra sus competencias para aplicar problemas del medio con este conocimiento (Sávio, D., Cândido Moraes, \& Motta Barreto, 2017).

Esta nueva forma de enseñanza es característica en la actualidad y responde a la adaptación permanente a los procesos de cambio del entorno (Luengo, Luzón, \& Torres, 2008), es una propuesta de formación efectiva dentro de los procesos de formación profesional, que incluye la adquisición de conocimientos con aproximación de realidades (González, Mieres, Denegri, \& Sepúlveda, 2014).

Conceptos tales como reingeniería y construcción sin pérdidas son ampliamente empleados en los proyectos de construcción. Si bien es cierto la reingeniería ha sido considerada una moda administrativa que nació hacia los 90 en Estados Unidos como estrategia para la documentación de información y que entró en desuso 
entre 1997 y 2007 debido a los desaciertos a nivel organizacional (Vergara-Schmalbach, Fontalvo, \& Morelos, 2013, p. 224), en los últimos años ha tomado vigencia por cuanto, justamente, se apoya en las lecciones aprendidas para el desarrollo de proyectos. En esencia, la reingeniería busca la revisión fundamental y el rediseño radical de los procesos productivos para alcanzar mejoras en rendimiento (Bustos, 2005, p. 3). Por lo anterior, desde la premisa de buscar la competitividad en las empresas constructoras, el reconocimiento de los aciertos y desaciertos del pasado puede ayudar a mejorar el proceso productivo en una obra.

En cuanto a la filosofía Lean, lo que se pretende es que se logre incremento de productividad, reducción de costos, mayor flexibilidad, mayor valor agregado al producto final y un incremento de resultados empleando un flujo de caja razonable con los materiales necesarios para aportar un producto de calidad (Raposo \& Cardoso Viera Machado, 2013), eliminando la superproducción, tiempos de espera y transportes innecesarios (Giroto \& Rodrigues, 2013). Según esto, Lean se centra en la materia prima, el producto, el proceso, el mercado y la forma de administrar (Flamarion, Petter, \& Valle Antunes Jr., 2008), todos estos conceptos relacionados estrechamente con el producto final de una obra civil.

Con el fin de responder a los nuevos retos derivados de la sociedad del conocimiento (Luengo, Luzón, y Torres, 2008) se desarrolla esta propuesta de actividades didácticas que tiene como objetivo afianzar conceptos relacionados con la administración de proyectos, fundamentando el ejercicio en estrategias de reflexión alrededor de reingeniería enfocada hacia la mejora en equipos y herramientas de construcción y Lean aplicado para el análisis de desperdicios en la actividad de mampostería, conceptos gerenciales que pueden influenciar en la calidad del producto a ser entregado en una obra. Aunque el planteamiento metodológico se centra en Administración de Obras, su aplicabilidad se extiende a los cursos de Métodos Constructivos y Proyectos de construcción. Además, si bien es cierto este planteamiento se centra en aplicaciones pedagógicas para Ingeniería Civil y Arquitectura, la estructura metodológica que la fundamenta puede orientarse hacia otras profesiones.

El enfoque de reflexión sobre diferentes etapas de procesos constructivos pretende que el futuro profesional analice aspectos significativos de estos conceptos y encuentre aplicación en su ámbito laboral (Arratia, 2008) proyectando su desempeño ocupacional en espacios en donde deba emplear sus conocimientos y habilidades en situaciones reales en la vida cotidiana (Iglesias \& Beltrán, 2012), pero también pueda desarrollar valores y actitudes (Palomer et al., 2013).

\section{Metodología}

Las estrategias de reflexión planteadas combinan las actividades curriculares y extracurriculares y los contenidos del curso Proyectos de Construcción e incorporan cuestionamientos que ubican al futuro profesional en su desempeño ocupacional con posturas ante diferentes situaciones. El diseño de las actividades se orientó para dar respuesta a las dificultades encontradas en la enseñanza de ciertos temas en este curso en tres cohortes (semestres consecutivos).

\section{Las estrategias pedagógicas de reflexión como apoyo a las pruebas del estado en Colombia}

Para el Consejo Nacional de Acreditación en Colombia el concepto de calidad en la educación superior está asociada al cumplimiento de características que posee la institución educativa y en virtud de las cuales se ofrece un servicio educativo en calidad óptima (Ministerio de Educación Nacional, 1998). El planteamiento de las estrategias pedagógicas basadas en reflexión que se presenta ha sido diseñado buscando que 
los futuros profesionales efectivamente adquieran los estándares de calidad requeridos en función de unas competencias de desempeño esperadas. Dichas competencias son evaluadas en los Exámenes de Estado de la Educación Superior en Colombia, hoy conocidos como Pruebas Saber Pro (Ministerio de Educación Nacional, 2010), exámenes que son de carácter obligatorio desde el 2009 (Ministerio de Educación Nacional, 2018).

En el taller de resultados que realizó el Ministerio de Educación Nacional se presentaron los resultados de estas pruebas que justamente contienen preguntas encaminadas a evaluar competencias generales y específicas empleando estrategias de pensamiento crítico, comunicación escrita, responsabilidad social entre otras modalidades (Ministerio de Educación Nacional, 2017). $\mathrm{Si}$ bien es cierto, las estrategias tradicionales pueden seguir siendo empleadas, la realidad es que estas no cumplen las expectativas que la educación del siglo XXI requiere (Sávio et al., 2017).

Lo anterior ratifica la necesidad de generar al interior del aula acciones pedagógicas que le permita al futuro profesional desarrollar buena capacidad comunicativa para relacionarse con el otro en un ambiente organizacional sano (Erren, 2008) y productivo (Bermúdez \& González, 2011) (Serrano Guzmán et al., 2017) que respete los aportes del otro y que aprenda a trabajar en equipo para alcanzar las metas (Ibarra \& Rodríguez, 2011); igualmente, que haga uso de su autonomía intelectual para entender su contexto (Vélez, 2012) (Serrano Guzmán et al., 2017) y responda de manera responsable en la formulación de soluciones técnicas que respeten los deberes solidarios de ciudad (Muñoz, 2011).

Las estrategias pedagógicas propuestas se han implementado en el curso de Proyectos de Construcción en el programa de Ingeniería Civil de la Pontificia Universidad Javeriana Cali y se encaminan hacia conceptos de construcción sin pérdida y reingeniería cuya apropiación le aporta al futuro egresado destrezas para el desempeño ocupacional en las obras en las que tenga opor- tunidad de trabajar. Sin embargo, la propuesta presentada puede ser implementada en diferentes programas educativos a nivel de pregrado y a nivel graduado en otras disciplinas.

\section{Resultados}

La reflexión ofrece un puente entre la teoría y la práctica bajo el entendido que "una onza de experiencia es mejor que una tonelada de teoría" (Bringle \& Hatcher, 1999). Las estrategias de reflexión fueron diseñadas para el curso Proyectos de Construcción partiendo de los lineamientos proporcionados en el Diplomado Aprendizaje+Servicio de la Pontificia Universidad Católica de Chile. Estas estrategias de reflexión permiten evaluar el aprendizaje de conceptos, desarrollar competencias/habilidades transversales como también actitudes y valores (Montalva, Ponce, \& Llorca, 2017), considerando competencias de pensamiento crítico, solución de problemas, compromiso social, trabajo en equipo y habilidades comunicativas. Justamente, si el futuro profesional desarrolla estas estrategias se podrá ofrecer a la industria y al medio laboral profesionales creativos y con espíritu de liderazgo (Serrano Guzmán et al., 2017; Shin et al., 2013: Oehrtman et al., 2010) cualidades y aptitudes que les permitirá defender sus posturas de manera crítica e interpretativa (Good \& McIntyre, 2015) en el marco de un ambiente ético (Vellurattil et al., 2014) y de responsabilidad social (Pérez de Maldonado, Bustamante, \& Maldonado, 2009). En el caso particular del curso Proyectos de Construcción, cada actividad se diseña considerando que debe permitir continuidad, estar relacionada con la temática abordada, constituirse en un reto para el estudiante y contextualizada a la realidad del grupo que la aborda (Montalva, Ponce, \& Llorca, 2017). Las partes fundamentales de la estrategia de reflexión incluyen el objetivo del curso, el objetivo que se pretende alcanzar con la actividad, recursos necesarios, duración, descripción de la actividad y anexos si es el caso. Estas estrategias están en consonancia con la estructuración de actividades en 
la metodología Aprendizaje+Servicio (Jouannet, Salas, \& Contreras, 2013) en donde, en algunos casos, intervienen socios comunitarios o beneficiarios. Se debe destacar también que estrategias de reflexión similares han sido planteadas en los cursos de Diseño I de pregrado y de Transporte de Contaminantes a nivel de posgrado. A continuación, se presentan las estrategias propuestas.

\subsection{Propuesta de reflexión en pensa- miento crítico}

Objetivo del curso: Identificar los conceptos contemporáneos de administración, organización y definición de un proyecto de construcción.

Objetivo de la estrategia de reflexión: Identificar situaciones del ejercicio ocupacional del ingeniero civil apoyadas en la costumbre y la implicación social que esto tiene en el desarrollo de una comunidad

Recursos: Lectura, computador, lápiz y papel

Modalidad de trabajo: individual y grupal

Duración: 30 min

Descripción: Los medios de comunicación constantemente informan sobre las repercusiones negativas para las comunidades ante la no ejecución de obras de infraestructura requeridas para la satisfacción de las necesidades básicas con el consecuente desmejoramiento de la calidad de vida de los habitantes.

Con esta actividad se identifican criterios personales para cambiar esta dinámica en el mundo. Se solicita a los alumnos que en $5 \mathrm{~min}$ lean el fragmento extractado de (Vega, 2015) "La leyenda del anillo de Giges", con el contenido que a continuación se transcribe textualmente:

Giges era un un pastor al servicio del rey de Lidia.... Un día, después de una tempestad y un terremoto, se agrietó la tierra, abriéndose un abismo en el lugar donde pastaba el rebaño de Giges. Asombrado, desciende en la grieta $y$ entre otras maravillas encuentra un caballo de bronce hueco con pequeñas aberturas. En el interior del mismo, había un cadáver de un hombre de gran tamaño que no llevaba sobre sí más que un anillo de oro en un dedo. Giges, tomó el anillo y se fue. Giges descubre que el anillo le confiere el poder de la invisibilidad y ante el beneficio de este, consigue que lo nombren pastor responsable de ir a rendir cuentas al rey. Después de mucho reflexionar, urdió un siniestro plan: ir al palacio, seducir a la reina, matar al rey y hacerse con el trono. Usando el privilegio de su anillo consigue materializar su plan, convirtiéndose en rey, pero no un rey cualquiera, uno tirano....Podría haber utilizado el anillo para descubrir por ejemplo a los criminales, para hacer una sociedad más justa, pero decide que es más interesante medrar adquiriendo riqueza y poder. Platón explica de esta manera la tiranía y corrupción de los dirigentes, al sentirse superiores, con poder e impunes y, cuestiona sobre 'que habríamos hecho cada uno de nosotros si hubiésemos encontrado el mágico anillo, distinguiendo entre expresar una opinión si se nos pregunta y ser dueños realmente del poder del anillo' (Vega, 2015).

En el libro II de la República se encuentra el conocido mito del anillo de Giges el cual muestra la noción de la injusticia humana y permite identificar el resurgimiento enérgico de la justicia (Higuera, 2016). La historia del anillo de Giges revela que portar el anillo le confiere confianza y libertad al pastor, con las cuales se transforma y le permiten hacer parte de un grupo social diferente (Higuera, 2016).

A continuación, se solicita que lean fragmentos tomados textualmente del escrito "El ser humano, la corrupción y la política" de Gerardo Berthin Siles en el volumen 5 de la revista Ciencia y Cultura (Berthin,1999) y que trata el tema de ética en política.

...[...]en política existe una ética diferente de la ética. Y se comprueba que con el pasar del tiempo el progreso en términos económicos y en otros términos ha resultado ser más importante que el desarrollo espiritual del ser humano, demostrando que existe una gran brecha entre lo que el hombre desea ser y lo que es en realidad, habría pues dos espacios, el del 
poder y el del no poder siendo el primero, el ámbito de lo político, el lugar donde el hombre miente, engaña, distorsiona, traiciona, de este modo se justificarían las guerras, por ejemplo. Para finalizar nos dice que el éxito político se mide si se puede mantener, incrementar o demostrar poder sobre otros, mientras que el éxito moral se mide si se puede demostrar en relación con los otros, que estos son objetivos en sí mismos (Berthin, 1999, p.1).

Luego, el docente orienta al grupo diciendo que se abre un espacio de discusión para que en plenaria los estudiantes respondan a las preguntas: 1) qué opina Ud. de la actitud de Giges? 2) en qué situaciones los ingenieros civiles pueden comportarse como Giges? 3) qué coincidencias pueden darse entre la actitud de Giges y la de aquellos que tienen el "anillo" de los fondos del Estado y son los encargados de administrar los fondos de los municipios en cada país? 4) de qué forma se puede ser partícipe activo en política manteniéndose en el marco de la ética y la moral? En esto, se destinan 20 minutos. Los resultados de la participación de los estudiantes se van registrando en el tablero.

Reflexión para el cierre de la actividad: Se finaliza haciendo énfasis en que la costumbre de "una vida de corrupción" debe desarraigarse del ejercicio ocupacional del ingeniero civil.

Evidencias de aplicación de esta estrategia de reflexión en otros cursos: el pensamiento crítico es una actividad reflexiva orientada a la acción (Zelaieta \& Camino Ortiz de Barrón, 2018) y puede aplicarse en diferentes ámbitos ya que permite analizar problemas de toda índole (France, de la Garza, Slade, Lafortune, Pallascio, \& Mongeau, 2003).

Este tipo ejercicios se han implementado en el curso Diseño I prerrequisito del curso Diseño II (Serrano et al., 2018), conducentes a grado a nivel de pregrado en Ingeniería Civil y en cursos de posgrado a nivel de maestría de Transporte de Contaminantes. En todo momento, para el desarrollo de esta actividad se busca un ambiente adecuado en el que los participantes puedan exponer sus argumentos generando una práctica comprometida con la transformación y para la mejora social (Zelaieta \& Camino Ortiz de Barrón, 2018).

\subsection{Propuesta de reflexión sobre com- petencia habilidades comunicativas}

Objetivos: Desarrollar habilidad de comunicación escrita; afianzar estrategias para la elaboración de resúmenes.

Recursos: Artículo de la web, computador con Word o procesador de texto.

Modalidad: Grupal, mínimo 2 y máximo 3 participantes.

Duración: 20 a 30 minutos.

Descripción: Teniendo en cuenta que el individuo emplea diferentes procesos y conocimientos de diversos tipos lingüísticos- sociolingüísticos para comunicarse, se deben fomentar estrategias para el fortalecimiento del manejo discursivo (Viera, 2010). En lo particular, en el curso de Proyectos de Construcción, el docente puede apoyarse en la construcción de definiciones como el concepto de la filosofía Lean aplicada a proyectos de construcción. Se procede a organizar los grupos de trabajo y se hace la siguiente invitación: "en un diálogo entre Uds. comenten lo que conocen acerca de Lean Manufacturing y en función de lo que conozcan, elaboren un párrafo que tenga entre 30 y 35 palabras". Se conceden 10 minutos.

Posteriormente, se solicita que cada grupo lea la definición que estructuró y se selecciona, por votación, la mejor definición. Como lo recomienda el Centro de Desarrollo Docente de la Pontificia Universidad Católica de Chile en la ventana Actividades de Reflexión $\mathrm{A}+\mathrm{S}$ : "ningún grupo puede votar por su propia definición" (Centro de Desarrollo Docente, 2017a). En esta actividad se destinan 5 minutos.

Una vez seleccionada la mejor definición, se solicita al grupo que se proceda a leer la cita textual del siguiente párrafo del artículo "El ingeniero industrial impactando el medio ambiente" 
de Ángela Patricia Anaya y Mario Fernando Acosta en la Revista Educación en Ingeniería (Anaya \& Acosta, 2010):

[...]Lean tiene como fundamentos el identificar las actividades de valor, entender el flujo de productos, servicios e información a través de la cadena de valor y entre los eslabones de la cadena de suministros, y, por último, la caracterización del desperdicio en las actividades de la empresa. [...] ]en las áreas de producción y manufactura los desperdicios son fácilmente identificables, mientras que, en el caso de información, el desperdicio es menos visible. La información es la que permite medir el desarrollo de las actividades para comparar con los estándares esperados o las metas fijadas y luego entonces entender si el funcionamiento del área, proceso o industria es el adecuado (Anaya \& Acosta, 2010, p. 182).

Y, que continúen con la lectura de la definición de Lean tomada y adaptada de (Raposo Rosa \& Cardoso Viera Machado, 2013):

Lean es una aproximación multidimensional que se extiende a grupos para gran variedad de prácticas administrativas que incluyen justo a tiempo, sistema de calidad, trabajo en equipo, producción por departamentos y administración de insumos en un sistema integrado. Cuando se logra la sinergia entre estos factores se logra alta calidad con bajos desperdicios (Raposo \& Cardoso Viera, 2013, p. 887).

Posteriormente, se indica al grupo que procedan a complementar la definición que se seleccionó con la definición aportada por Anaya \& Acosta (2010) y de Raposo \& Cardoso Viera (2013) y que, finalmente, escriban un párrafo que contenga por lo menos dos oraciones que resuman lo que podría ser la definición de la
Filosofía Lean aplicada a la construcción. Se solicita además que la nueva definición no exceda 60 palabras y que como mínimo tenga 55.

Reflexión para el cierre de la actividad: Se finaliza el ejercicio invitando a los participantes que señalen las dificultades encontradas para elaborar la definición.

Evidencias de aplicación de esta estrategia de reflexión en otros cursos: Esta actividad se adelantó con estudiantes del curso Diseño I, empleando el concepto de vivienda de interés social rural. El ejercicio permitió incluir en la definición de vivienda el concepto de sostenibilidad el cual no estaba inicialmente considerado.

\subsection{Propuesta de reflexión sobre com- petencia solución de problemas}

Objetivos: Identificar causas de desperdicios en actividad de mampostería; plantear alternativas para el aumento de la productividad en mampostería.

Recursos: Visita de campo y lectura de manuales para el diligenciamiento de proyectos con la Matriz General Ajustada (MGA) del Departamento Nacional de Planeación.

Modalidad: Grupal, entre 2 y 3 integrantes.

Duración: 40 minutos.

Descripción: Esta actividad se realiza luego de la visita de campo. El docente orienta a los estudiantes sobre la importancia de la observación para identificación de factores que afectan el rendimiento en la actividad de mampostería. En la visita de campo, el grupo de trabajo debe registrar las causas de la pérdida de materiales y de tiempo en la actividad de mampostería en una empresa constructora. Se elabora el árbol de causas y efectos en una infografía similar a la Figura 1. 


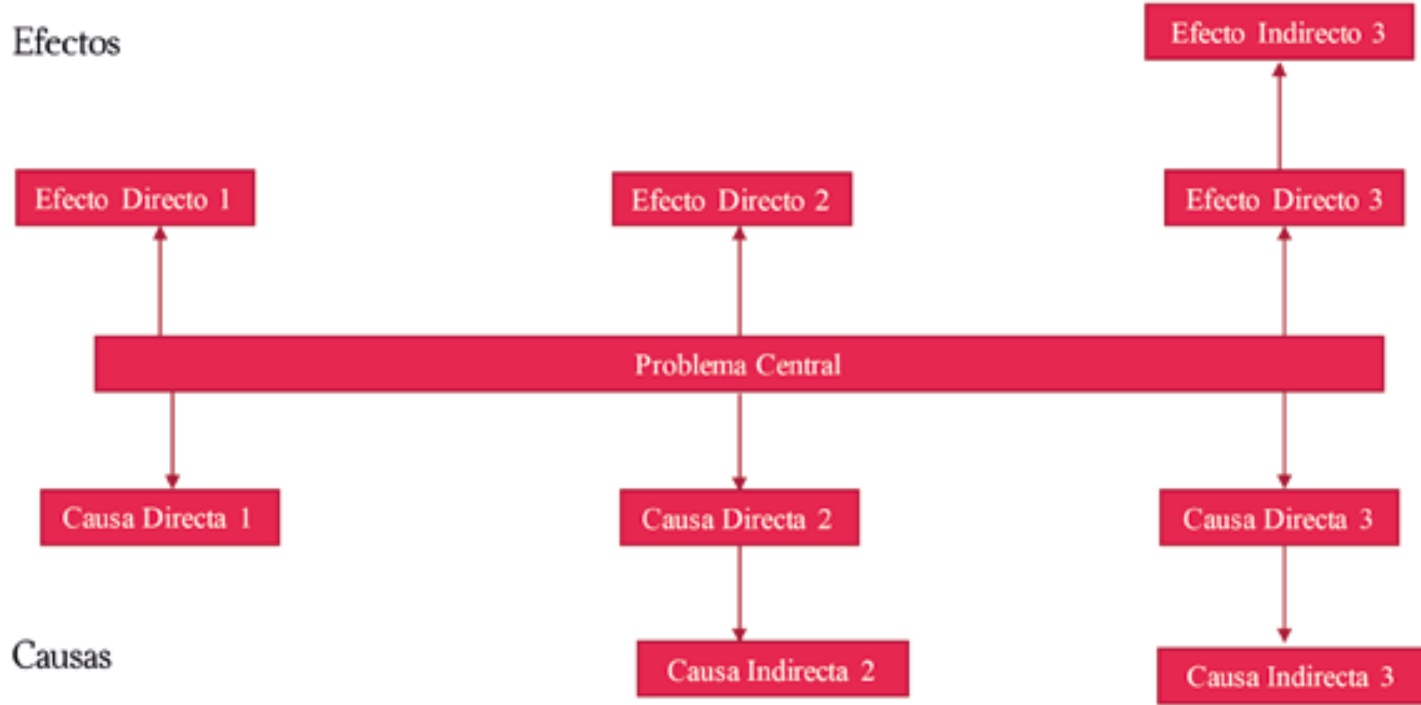

Fuente: Autores con información adaptada de Subdirección Territorial y de Inversiones Públicas del Departamento Nacional de Planeación, 2013.

Se espera que el estudiante genere un Figura 2. El tiempo destinado para esta actividad árbol de problemas similar al indicado en la son $15 \mathrm{~min}$.

Figura 2. Árbol de problemas identificados durante la visita

Efectos

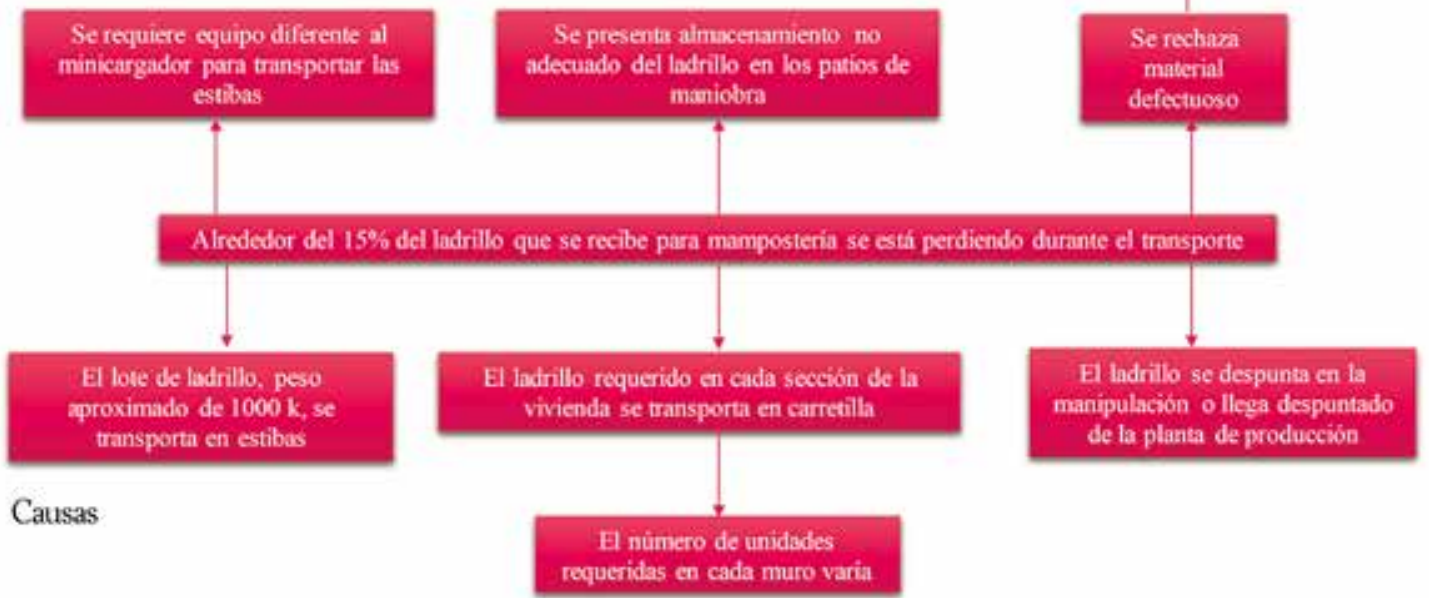

Fuente: Autores con información adaptada de Bejarano (2017). 
En cada grupo de trabajo, se plantea el árbol de objetivos, con sus correspondientes fines y medios según lo establece la metodología MGA. Tiempo de dedicación: 30 min.
El Árbol de Objetivos es propositivo al árbol de problemas y permite cerrar conceptualmente el proyecto en análisis (Figura 3).

Figura 3. Árbol de objetivos o de fines y medios

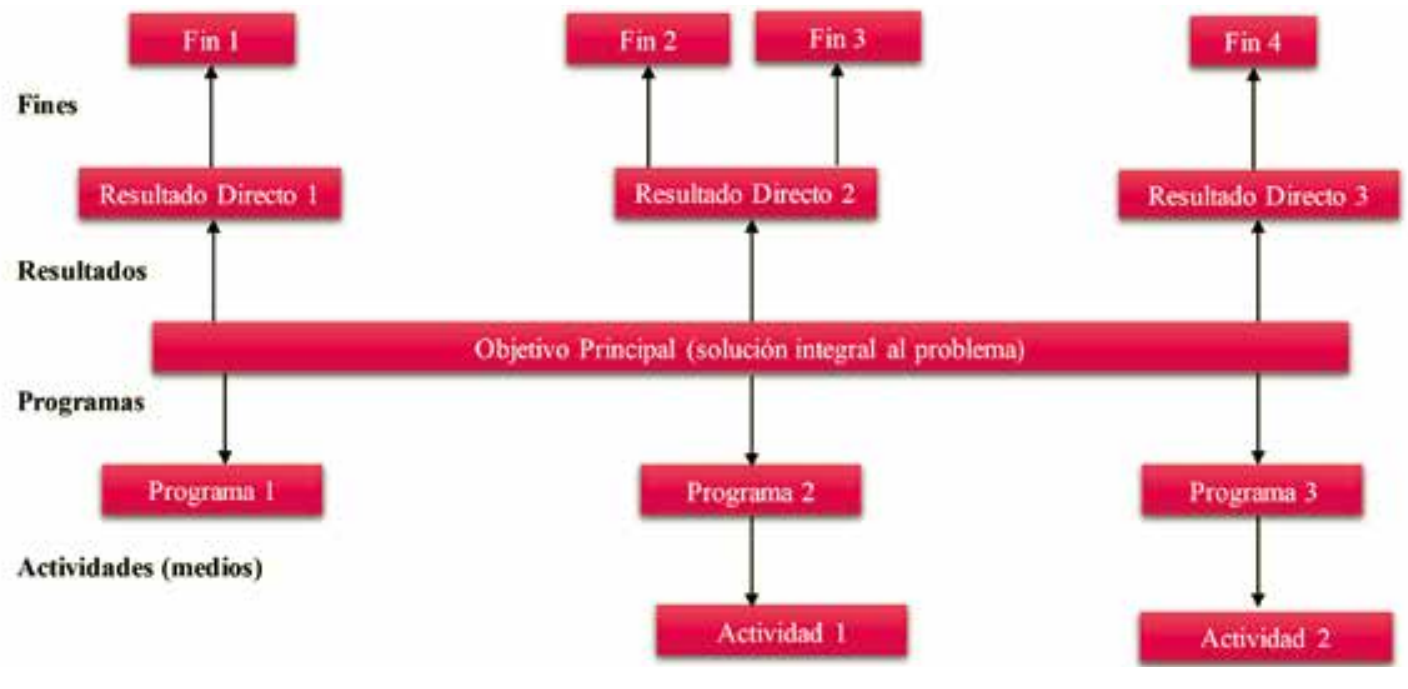

Fuente: Autores con información adaptada de Bejarano (2017).

Se espera que el estudiante genere un árbol

El tiempo destinado a esta etapa son $15 \mathrm{~min}$. de objetivos similar al indicado en la Figura 4.

Figura 4. Árbol de fines medios

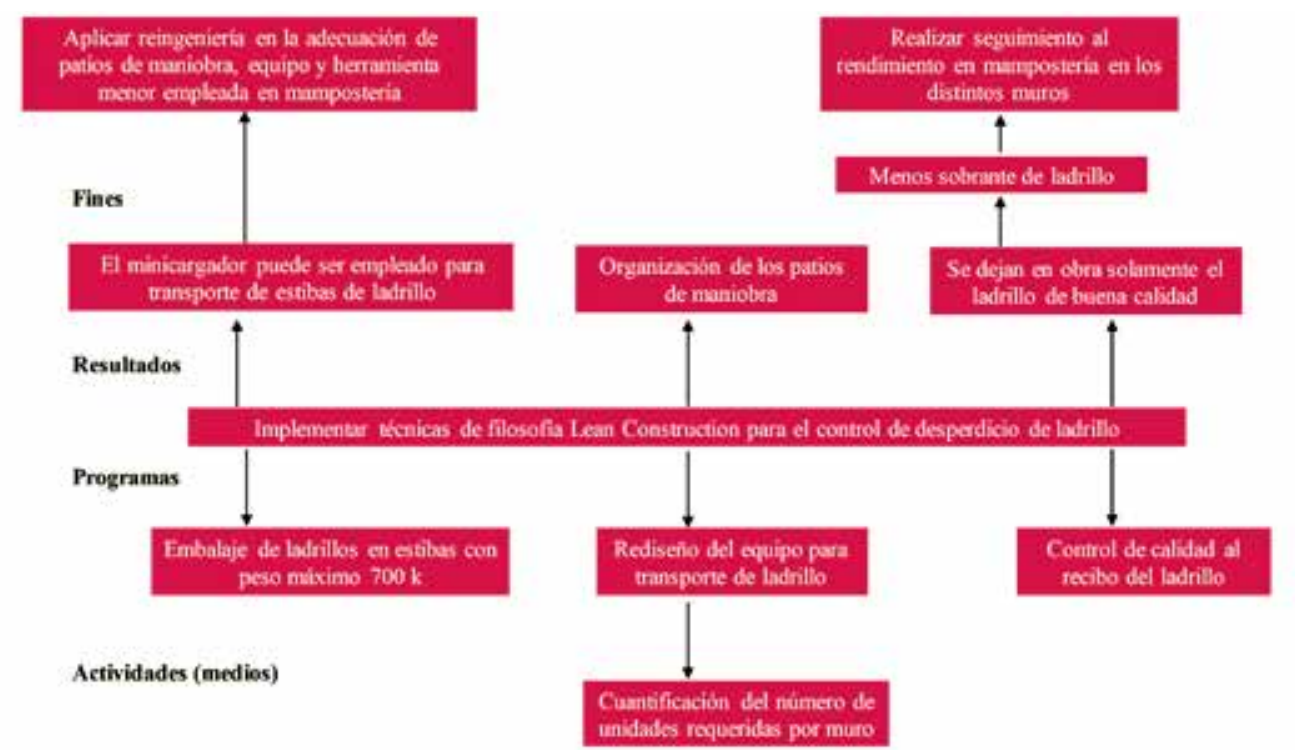

Fuente: Autores con información adaptada de Bejarano (2017). 
Reflexión para el cierre de la actividad: Se finaliza el ejercicio solicitando al grupo de estudiantes que evalúen los beneficios que acarrea la implementación de un programa de construcción sin pérdidas en otras actividades de una obra. Así mismo, que señalen las dificultades técnicas y de recurso humano a las que probablemente pudiera enfrentarse el equipo de trabajo que acomete estas actividades. Al respecto, esta actividad de reflexión le permite al estudiante aprender mediante la interacción social y práctica que recopila las vivencias de una obra lo que le permite percibir y transformar la realidad (González, et al., 2014) y adaptar lo que observa para un mejoramiento de los procesos de construcción.

Evidencias de aplicación de esta estrategia de reflexión en otros cursos: Esta actividad se ha implementado en el curso de Diseño I en donde el estudiante finalmente aprende a priorizar inversiones (Serrano et al., 2018). La experiencia adelantada en el segundo semestre de 2017 involucró a cuatro grupos de estudiantes que partieron de una información existente de comunidades del país e identificaron los proyectos que pudieran satisfacer las necesidades de los habitantes.

\subsection{Propuesta de reflexión sobre com- petencia compromiso social}

Objetivos: Propiciar espacios de reflexión sobre la importancia de modificación o cambios radicales en herramientas y equipos de construcción con miras a la implementación de la filosofía Lean en actividades de construcción; sensibilizar a los estudiantes sobre la responsabilidad social del ingeniero frente al recurso humano.

Recursos: imágenes de herramienta y equipos empleados en proyectos de construcción.

Modalidad: Grupal, entre 2 y 3 personas

Duración: 40 minutos

Descripción: La actividad consiste en profundizar sobre el concepto de reingeniería aplicada en equipos y materiales de construcción empleados en diferentes procesos en una obra.
Se parte de una lectura de un fragmento citado textualmente de (Vergara-Schmalbach, Fontalvo Herrera, \& Morelos Gómez, 2013, p. 224), en donde definen el concepto de reingeniería:

...Reingeniería es la mejora radical de los procesos con resultados de gran impacto a diferencia de otros enfoques que se caracterizan por buscar resultados incrementales y continuos... (Bustos, 2005, p. 3). [...] ...Como tendencia administrativa, la reingeniería se está centrando en la actualidad en nuevos desarrollos de proyectos basándose en el estudio de los errores y éxitos pasados (Vergara-Schmalbach, Fontalvo, \& Morelos, 2013, p. 224).

Posteriormente, se invita a los estudiantes a revisar las imágenes de la Tabla 1 y a identificar los cambios radicales que ocurrieron para llegar a las adecuaciones en los elementos constructivos.

Reflexión para el cierre de la actividad: El profesor insta al grupo a elaborar el análisis de precios unitarios de cada uno de los cambios ejecutados y a estimar el tiempo de vida útil de los elementos constructivos presentados y los posibles riesgos de los trabajadores.

Evidencias de aplicación de esta estrategia de reflexión en otros cursos: Esta estrategia se ha adaptado para estudiantes de nuevo ingreso a quienes se les plantean que identifiquen condiciones de escenarios propicios para la accidentalidad al interior de la obra. Lo anterior porque se considera que el ingeniero debe estar comprometido en defender la salud ocupacional y propiciar condiciones para mitigar los riesgos laborales que, aunque son acciones individuales que le competen al trabajador, el efecto para el normal desarrollo de las obras implica actuaciones de todos y cada uno de los miembros de una institución, en este caso de una obra (Guarro, 2005).

\subsection{Propuesta de reflexión sobre com- petencia trabajo en equipo}

Objetivo: Afianzar el conocimiento del grupo de trabajo sobre la organización en obra para la 
implementación de la filosofía Lean Construction en la actividad de mampostería.

Recursos: Papel y computador

Modalidad: Grupal, mínimo 2 y 3.

Duración: 50 min.

Descripción: Esta reflexión busca ser un mecanismo para que el grupo revise los aprendizajes logrados como grupo (Centro de Desarrollo Docente, 2017b) y se debe realizar luego de la visita a la obra. Se puede contar con la presencia de un funcionario de la obra visitada.

Se procede a realizar la lectura de los siguientes hechos encontrados en tres obras. La obra 3 se ejecutó luego de finalizada la obra 2 y la obra 2 se ejecutó una vez finalizada la obra 1.

Obra 1 (522 unidades de vivienda): El ladrillo inicialmente se entregaba en pacas de 1000 $\mathrm{kg}$ actualmente, es estibado en pacas de $700 \mathrm{~kg}$. Se procedió a generar la práctica de recolección de ladrillo despicado, fisurado o con alguna imperfección, para ser utilizado como cortes de medios, cortes especiales, culatas o se modifica según la modulación en piezas utilizables. Se hicieron modulaciones en cortes de ladrillo y se veló por la nivelación del área sobre la cual se hacía la entrega del material. Se clasificaron los mamposteros según su nivel de experticia en ciertos muros.

Obra 2 (968 unidades de vivienda): Con el aprendizaje de la obra anterior, se aplicaron las mismas estrategias, con las siguientes mejoras:
- Se asignaron dos personas encargadas constantemente de la recolección de sobrantes y desperdicios.

- Se implementaron penalidades por generación de desperdicios.

- La nivelación de los sitios de acopio de ladrillo se planificó desde el inicio del movimiento de tierra.

- La ladrillera aceptó recibir el material con imperfectos.

Las anteriores acciones tuvieron como consecuencia que se disminuyera el porcentaje de desperdicio del ladrillo medio estructural del $5 \%$ al $2 \%$.

Obra 3 (565 unidades de vivienda): adicional a lo ejecutado en las obras 1 y 2 , se realizaron los siguientes ajustes:

- Se implementó el ladrillo estructural con perforación vertical en lugar del ladrillo farol.

- Se acondicionaron los patios de maniobra para que el ladrillo viga-bloque quedara cerca de la cortadora.

- Se programó la entrega de ladrillo según programación de la obra.

\section{Tabla 1. Reingeniería en mejoramiento de los tableros eléctricos}

\begin{tabular}{|l|l|l|l|}
\hline \multirow{2}{*}{ Elemento } & \multicolumn{2}{c|}{ Características } & Cambios entre los elementos \\
\cline { 2 - 5 } & Costo & Número de usos & Entre 1 y 2 \\
\hline & & & \\
\hline
\end{tabular}




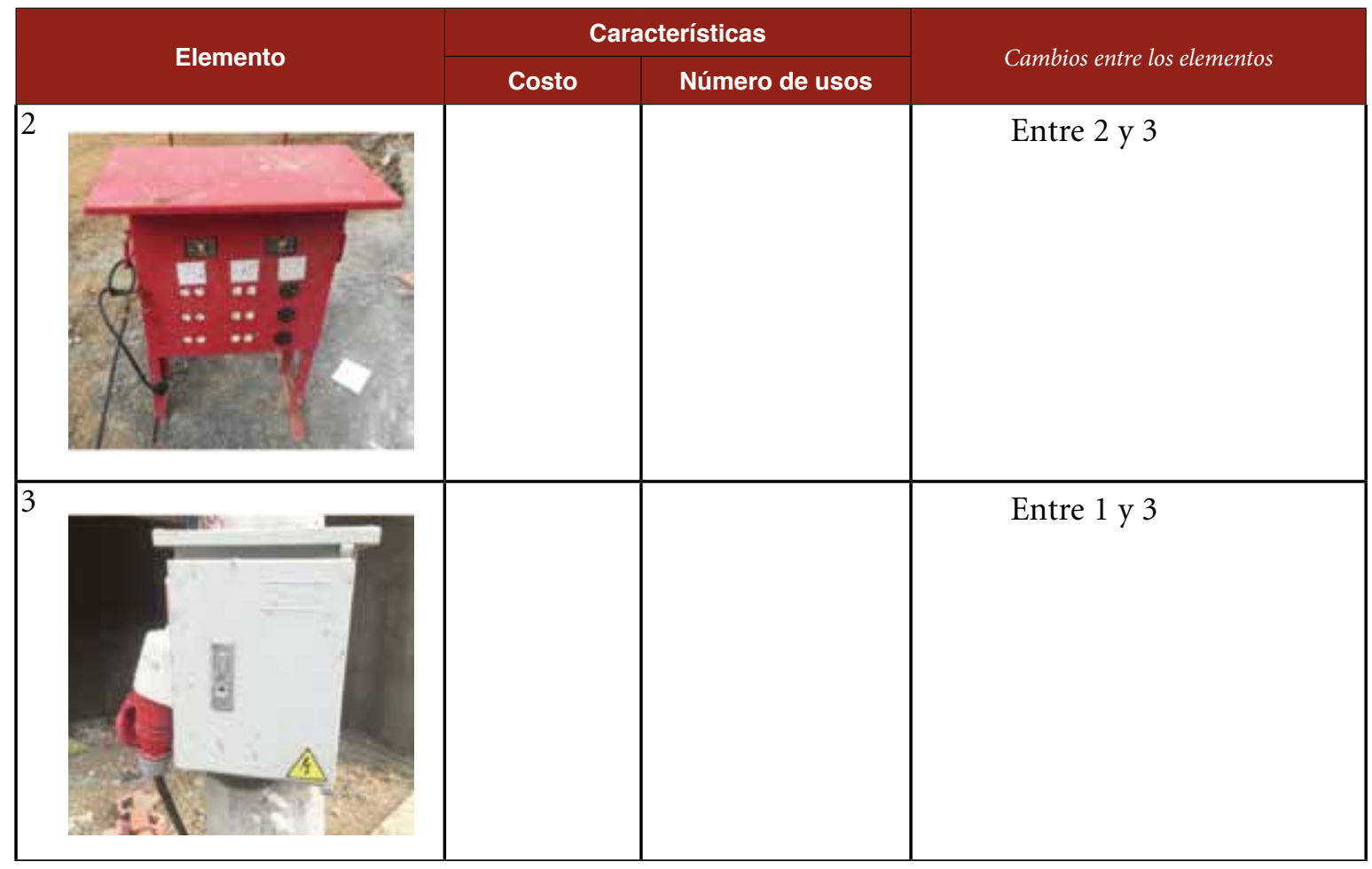

Fuente: autores partiendo de información de Bejarano (2017).

En la Tabla 2 se encuentran diferentes actividades asociadas a la mampostería. El equipo de trabajo debe organizar las actividades de la forma que garantice el cumplimiento de aspectos de Lean que fueron implementados en las tres obras para el proceso de mampostería.

Reflexión para el cierre de la actividad: Esta actividad se acompaña de una rúbrica de evaluación que recoge la coevaluación del equipo de trabajo.

El docente puede aprovechar el espacio para reflexionar sobre la relevancia de la distribución de funciones en un grupo para el buen logro de los objetivos y de la importancia de definir las funciones (roles) en una obra.

Evidencias de aplicación de esta estrategia de reflexión en otros cursos: Esta estrategia se ha empleado en Diseño I, curso prerrequisito de Diseño II y este a su vez, requisito para culminación del programa de ingeniería civil. Durante el desarrollo del programa se observó complementariedad en los equipos de trabajo, cumplimiento del cronograma de actividades para la ejecución de los proyectos del $98 \%$ y el tres de los cuatro grupos realizaron la coevaluación de sus pares de manera ecuánime. 
Tabla 2. Actividades ejecutadas para la labor de mampostería

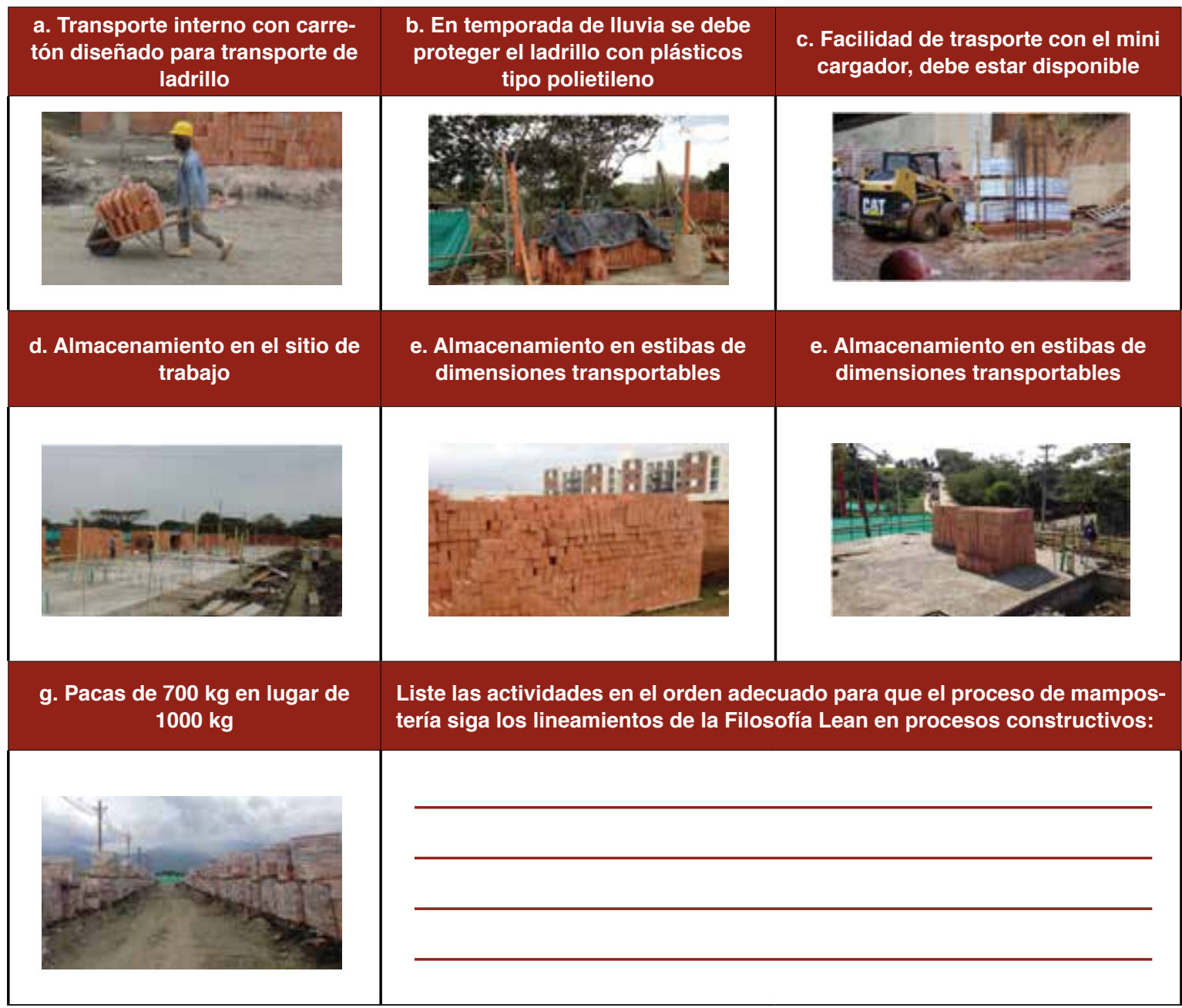

Fuente: Autores con información de Bejarano (2017).

\section{Conclusiones}

La comprensión de los procesos constructivos y las implicaciones que tiene en la calidad del producto final a ser entregado en un proyecto de construcción son factores claves para que el cliente o el usuario final sienta que el producto, bien o servicio entregado satisface completamente sus expectativas. Las estrategias de reflexión presentadas son adaptables para la enseñanza de la administración de recurso humano y de materiales bajo las modalidades de aplicación de rein- geniería y la implementación de Lean entendiendo, además, que esta filosofía de construcción sin pérdidas aporta beneficios al constructor, pero principalmente al ambiente porque se disminuyen desperdicios en materiales. Las estrategias de reflexión propuestas sirven para el desarrollo de competencias como solución de problemas, habilidades comunicativas, compromiso social, pensamiento crítico y trabajo en equipo, que en conjunto le permiten al futuro profesional emplear sus conocimientos sobre aspectos gerenciales a la solución de problemáticas que pueden 
presentarse en una obra. El diseño de las estrategias y su aplicación a la enseñanza de la ingeniería civil, específicamente a contenidos sobre proyectos de construcción, promueve la implementación de prácticas reflexivas que consideren la formación sólida, ética y solidaria del futuro profesional y la responsabilidad del ejercicio durante su desempeño ocupacional en la construcción de una sociedad justa y democrática. Se espera que estas estrategias respondan a las exigencias de la competitividad globalizada que de manera insistente exige cambios a las instituciones de educación superior durante la formación de los futuros profesionistas.

\section{Referencias bibliográficas}

Anaya, A., \& Acosta, M. (junio de 2010). El ingeniero industrial impactando el medio ambiente. Revista Educación en Ingeniería, 5(9), 179187. Recuperado de https://goo.gl/ZRLYxb

Arratia Figueroa, A. (2008). Ética, solidaridad y "Aprendizaje Servicio" en Educación Superior. Acta Bioethica, 14(1), 61-67. http://dx.doi.org/10.4067/S1726-569X2008000100008

Bejarano, A. (2017). Prácticas constructivas en proyectos de vivienda en la Constructora Jaramillo Mora S.A. Cali: Archivo de la empresa Jaramillo Mora S.A.

Bermúdez, L., \& González, L. (enero-junio de 2011). La competencia comunicativa: elemento clave en las organizaciones. Quórum Académico, 8(15), 95-110.

Berthin Siles, G. (junio de 1999). El ser humano, la corrupción y la política. Revista Ciencia y Cultura, 5, 111-120.

Bringle, R., \& Hatcher, J. (1999). Reflection in servicelearning: making meaning of experience. Educational Horizons, 179-185.

Bustos, C. (2005). La reingeniería: herramienta controversial. Visión Gerencial, 1, 3-10. Recuperado de https://goo.gl/r2VE7u

Centro de Desarrollo Docente (12 de octubre de 2017a). Aprendizajes del equipo. Campus San Joaquín, Chile: Pontificia Universidad Católica de Chile. Recuperado de https://goo.gl/NaC2ft

Centro de Desarrollo Docente (2017b). Mitad y mitades. Campus San Joaquín: Pontificia
Universidad Católica de Chile. Recuperado de https://goo.gl/yhnuH5

Erren, M. (2008). La competencia comunicativa del orador. Acta Poética, 28(1), 121-156.

Flamarion Klippel, A., Petter, C., \& Valle Antunes Jr., J. (marzo de 2008). Lean management implementation in mining industries. Dyna, 75(154). Recuperado de https://goo.gl/HjkcL3

France, D., de la Garza, M., Slade, C., Lafortune, L., Pallascio, R., \& Mongeau, P. (2003). Qué es el pensamiento dialógico crítico? Perfiles Educativos, XXV(102), 22-39.

Giroto Rebelato, M., \& Rodrigues, A. (2013). As contradicoes do Lean Sig Sigma. Revista de Administração da Unimep, 11(2), 57-81. Recuperado de https://goo.gl/HrFmKx

Good, D. J., \& McIntyre, C. (2015). Use of journal clubs within senior capstone courses: Analysis of perceived gains in reviewing scientific literature. Journal of Nutrition Education and Behavior, 47(5), 477-479. doi: https://doi. org/10.1016/j.jneb.2015.05.003

González Gómez, Y., Mieres Chacaltana, M., Denegri Coria, M., \& Sepúlveda Aravena, J. (septiembre-diciembre de 2014). La enseñanza de nociones básicas de economía en la formación docente. Una propuesta desde la experiencia de dos instituciones de Educación Superior Regional. Profesorado: Revista de currículum y formación del profesorado, 18(3), 411-430.

Guarro Pallás, A. (2005). La transformación democrática de la cultura escolar: una respuesta justa a las necesidades del alumnado de zonas desfavorecidas. Profesorado, revista de currículum y formación del profesorado, 1(1), 1-48.

Higuera Linares, M. (julio-diciembre de 2016). El mito del anillo de Giges en la República de Platón. Universitas Philosophica, 33(67), 73-102. Recuperado de https://goo.gl/8Ez6Bv

Ibarra Sáiz, M., \& Rodríguez Gómez, G. (2011). Aprendizaje autónomo y trabajo en equipo: reflexiones desde la competencia percibida por los estudiantes universitarios. Revista electrónica interuniversitaria de Formación del profesorado, 14(4), 73-85.

Iglesias Rodríguez, A., \& Beltrán Llavador, F. (2012). Practicum sin fronteras: Estudio de un caso 
de reflexión intercultural y pedagógica. Teoría de Educación, 24, 105-131.

Jouannet, C., Salas, M., \& Contreras, M. (2013). Modelo de implementación de Aprendizaje Servicio $(A+S)$ en la UC: Una experiencia que impacta positivamente en la formación profesional integral. Calidad en la Educación, 39, 197-212.

Luengo Navas, J., Luzón Trujillo, A., \& Torres Sánchez, M. (2008). Las reformas educativas basadas en el enfoque por competencias: una visión comparada. Profesorado: revista del currículum y formación del profesorado, 12(3), 1-10.

Ministerio de Educación Nacional (febrero de 1998). Consejo Nacional de Acreditación Lineamientos para la Acreditación. Bogotá: Ministerio de Educación Nacional.

Ministerio de Educación Nacional (27 de octubre de 2010). Las Pruebas Saber Pro (antes Ecaes), una ventaja competitiva en el campo laboral. Bogotá. Recuperado de https://goo. $\mathrm{gl} / 4 \mathrm{C} 2 \mathrm{rxU}$

Ministerio de Educación Nacional (18 de julio de 2017). Divulgación 2017 de Saber Pro. Bogotá. Recuperado de https://goo.gl/NHKDqD

Ministerio de Educación Nacional (15 de enero de 2018). Portal Colombia Aprende. Recuperado de https://goo.gl/W4MzQz

Montalva, J., Ponce, C., \& Llorca, C. (4 de noviembre de 2017). Elementos claves para el diseño de una reflexión de calidad en Aprendizaje Servicio. Lectura obligatoria en el Diplomado Aprendizaje+Servicio de la Pontificia Universidad Católica de Chile. Santiago de Chile: Centro de Desarrollo Docente de la Pontificia Universidad Católica de Chile.

Muñoz Gaviria, D. (enero-junio de 2011). El compromiso social de las facultades de educación: Reflexiones pedagógicas en torno a la educación y la crisis de la modernidad. El Ägora USB, 11(1), 125-152.

Oehrtman, S. J., Smolen, D., Hoblet, K. \&, Phillips, K. A. (2010). The comprehensive examination: A viable master's of science in nursing capstone course. Journal of Professional Nursing, 26(6), 360-365. doi: https://doi. org/10.1016/j.profnurs.2010.08.003

Palomer, L., Humeres, P., Sánchez, A., González, S., \& Contreras, A. (junio de 2013). Una experien- cia de "aprendizaje-servicio": fomentando el desarrollo de valores en estudiantes de odontología chilenos. FEM: Revista de la Fundación Educación Médica, 16(2), 91-96. http://dx.doi.org/10.4321/S2014-98322013000200007

Pérez de Maldonado, I., Bustamante Uzcátegui, S., \& Maldonado Pérez, M. (2009). El trabajo en equipo y el desarrollo de habilidades conversacionales en organizaciones educativas. Omnia, 15(3), 78-96.

Raposo Rosa, A., \& Cardoso Viera Machado, M. (2013). Lean Accounting: Accounting contribution for Lean Management Philosophy. Tourism y Management Studies, 3, 886-895. Recuperado de https://goo.gl/fWTfgp

Sávio Giordani, D., Cândido Moraes, E. d. J., \& Motta Barreto, M. A. (2017). Simulation of a competitive business environment: a case study in a Chemical Engineering Program. Production, 24(27), 1-12.

Serrano Guzmán, M., Pérez Ruiz, D., Torrado Gómez, L., \& Solarte Vanegas, N. (mayo-agosto de 2017). Consideraciones académicas y administrativas para implementación de Capstone en ingeniería civil: Estudio de caso. Revista Electrónica Educare, 21(2), 1-22. http://dx.doi.org/10.15359/ree.21-2.17

Serrano Guzmán, M., Pérez Ruiz, D., Torrado Gómez, L., \& Solarte Vanegas, N. (2018). Estrategias de reflexión en trabajo final requisito para grado: estudio de caso en Ingeniería Civil. Revista Dilemas Contemporáneos: Educación, Política y Valores, 3(15), 1-24, mayo.

Shin, Y.-S., Lee K-W., Ahn, J-S. \& Jung, J-W. (2013). Development of Internship \& Capstone Design Integrated Program for UniversityIndustry Collaboration. Procedia - Social and Behavioral Sciences, 102, 386-391. doi: https://doi.org/10.1016/j.sbspro.2013.10.753

Subdirección Territorial y de Inversiones Públicas del Departamento Nacional de Planeación. (2013). Prototipo Proyectos Productivos Metodología General para la Formulación y Evaluación de Proyectos de Inversión Pública- MGA, Sistema general de Regalías SGR, Versión 1.0. Bogotá. Recuperado de https://goo.gl/BvWKHM

Ucol-Ganiron Jr. T., \& Alaboodi, A. S. (2013). Cultural Learning Environment in Structural 
Engineering Courses of Architecture and Civil Engineering Students in Qassim University. Procedia - Social and Behavioral Sciences, 102, 300-310.

Vega, G. (23 de Mayo de 2015). La ética. La leyenda del anillo de Giges. Valladolid. Recuperado de https://goo.gl/aBCnQR

Vélez Gutiérrez, C. (julio-diciembre de 2012). Una reflexión interdisciplinar sobre el pensamiento crítico. Revista Latinoamericana de estudios educativos, 9(2), 11-39.

Vergara-Schmalbach, J., Fontalvo Herrera, T., \& Morelos Gómez, J. (septiembre-diciembre de 2013). ¿Es la ingeniería una moda administrativa? Revista Virtual Universidad Católica del Norte, 40, 216225. Recuperado de https://goo.gl/dHtnnj
Vellurattil, R. P., Puglisi, M. P., Johnson, C. L., \& Slonek, J. (2014). Introduction of a capstone research program in a new college of pharmacy: Student perceptions. Currents in Pharmacy Teaching and Learning, 6(3), 429-436. https://doi.org/10.1016/j.cptl.2014.02.008

Viera Bayeaux, I. (abril-junio de 2010). Algunos criterios acerca de la competencia comunicativa. EduSol, 10, 29-38.

Zelaieta Anta, E., \& Camino Ortiz de Barrón, I. (enero-marzo de 2018). El desarrollo del pensamiento crítico en la formación inicial del profesorado: análisis de una estrategia pedagógica desde la visión del alumnado. Profesorado: revista de currículo y de formación del profesorado, 22(1), 197-214. 